\title{
Using Machine Learning to optimize energy consumption of HVAC systems in vehicles
}

\author{
Martin Böhme ${ }^{1}$, Andreas Lauber ${ }^{1}$, Marco Stang ${ }^{1}$, Luyi Pan ${ }^{1}$ and Eric Sax ${ }^{1}$ \\ ${ }^{1}$ Karlsruhe Institute of Technology, \\ Institute for Information Processing Technologies (ITIV), \\ Engesserstraße 5, 76131 Karlsruhe, Germany \\ \{martin.boehme, andreas.lauber, marco.stang, eric.sax\}@kit.edu
}

\begin{abstract}
The detachment and calculation of functionalities from a vehicle into a cloud creates new chances. By linking different data sources with the invehicle data in the cloud, an optimization of these functionalities in terms of energy efficiency can be applied. For example, the Heating, Ventilation and Air Conditioning (HVAC) consumes up to $30 \%$ of total energy in a vehicle. Electric vehicles in particular lead to these high values because they are not able to recover the waste heat from combustion engines for interior heating. Therefore, the optimization of energy efficient strategies with respect to the vehicle energy management system becomes more relevant. Forecasts of the interior vehicle temperature are directly related to the HVAC energy consumption. This work focuses on the implementation and accuracy evaluation of Recurrent Neural Networks $(\mathrm{RNN})$ for interior vehicle temperature forecasting.
\end{abstract}

Keywords: Heating Ventilation and Air Conditioning (HVAC) - Energy Efficiency $\cdot$ Internet of Things $\cdot$ Machine Learning

\section{Introduction}

The integration of vehicles with the Internet of Things (IoT) enables a stream of relevant sensor data into a cloud system [1]. The combination of different external data (e.g. weather forecasts, traffic conditions) with in-vehicle data in the cloud allows new strategies for the energy optimization of the HVAC system. Forecasts of the interior vehicle temperature enables the creation of optimized HVAC control (e.g. On/Off schedules). The resulting system predicts future peak loads and preconditions the vehicle according to the created schedules. Such forecasting problems have been an issue of great importance especially for the case of building HVAC systems [2]. Machine learning algorithms allow us to tackle these problems in a novel way as they are able to learn and predict patterns based on different inputs. 


\section{State of the Art of Time Series Forecasting Methods}

\subsection{Recurrent Neural Networks}

In feed-forward neural networks, outputs of one layer is fed as input into the subsequent layers and each unit does relatively simple computations. Outputs are independent of each other i.e., output at time $t$ is independent of output at $t-1$. This idea of independence considered above does not match with sequences such as time-series data which consists of short-long term temporal dependencies that should also be taken into account [3]. For any given sequential data, the output depends upon previous information as well as the current input as shown in the Architecture of RNNs in Figure 1.

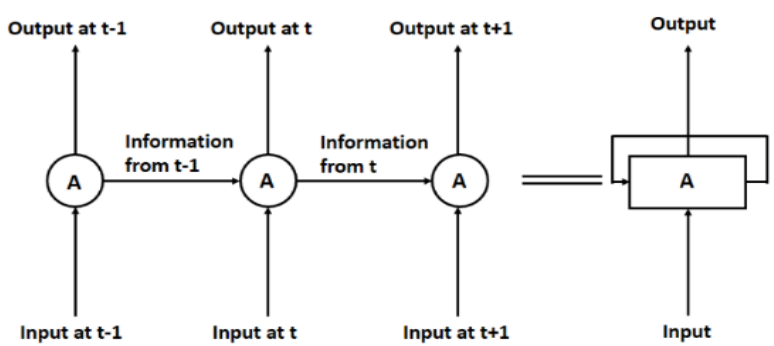

Fig. 1. Architecture of Recurrent Neural Network Model [3]

Still RNNs lag in learning as the gap between required previous information and the point of requirement increases to a large extent.

\subsection{Long Short Term Memory Networks}

LSTM is a special architecture of a recurrent neural network especially used in the field of deep learning. The LSTM contains special units called memory blocks in the recurrent hidden layer. The memory blocks contain memory cells with selfconnections storing the temporal state of the network in addition to special multiplicative units called gates to control the flow of information [4].

A schematic of a LSTM block can be seen in Figure 2. It features three gates (input, forget and output), block input, a single cell (the constant error carousel), and an output activation function. The output of the block is recurrently connected back to the block input and all of the gates [5]. 


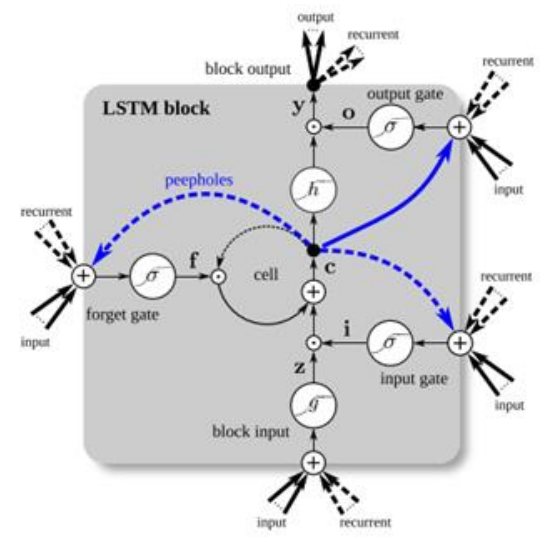

Fig. 2. Detailed schematic of a LSTM block [5]

A schematic of a LSTM block can be seen in Figure 2. It features three gates (input, forget and output), block input, a single cell (the constant error carousel), and an output activation function. The output of the block is recurrently connected back to the block input and all of the gates [5].

\section{Concept for vehicle HVAC optimization}

Modern vehicles are equipped with numerous sensors that create huge amount of data transmitted over the vehicle`s bus communication systems [6]. Modern digital communication paves the way to send this data from Controller Area Network (CAN) and other bus systems out of the vehicle into a cloud (V2X). As the cloud is basically a high-performance computer it is able to handle computing-intensive tasks like machine learning. We want to use this data to perform machine learning algorithms in the cloud and return the calculation results back into the vehicle.

In order to optimize the energy consumption of a HVAC system, vehicle sensor data correlated to the operation of the system needs to be streamed into the cloud. The control of HVAC systems is calculated based on different temperature or temperature correlated inputs. The data we used for our machine learning forecasts is described in the following chapter.

\section{$4 \quad$ Datasets and Setup}

The Living Lab Bus Project ${ }^{1}$ offers development opportunities utilizing the fleet of electric buses running in normal operation on a few of Helsinki Region Transport (HSL) lines. The buses serve as a mobile sensor platform providing extensive selection of real-time and accumulated history data from both existing and third-party de-

\footnotetext{
${ }^{1}$ Living Lab Bus project: http://livinglabbus.fi/
} 
vices. We used the data from the sensors that were installed on the bus. The sensor data is measured with a sampling rate of $T=1 \mathrm{~s}$. However, in order to create better forecasting models, each time series is sub-sampled with a period of $T^{\prime}=5 \mathrm{~min}$.

Three sensor signals were taken into consideration:

- Interior temperature in degrees Celsius. This is the interesting forecasting variable.

- $\quad$ Air pressure measured in $\mathrm{kPa}$

- Humidity in mass of water vapor $\mathrm{mH} 20$

The data handled needed further preparation as there were missing datapoints and busses didn't have the same operating times each day. The training and testing dataset were created with consecutive days, where each day included the same operating times.

Table 1 displays the training and testing dataset used for further processing.

Table 1. Description of Training and Testing Datasets

\begin{tabular}{|l|l|l|l|}
\hline Dataset & Number of Days & Operating time & Time interval \\
\hline Training & 34 & 12 hours & $5 \mathrm{mins}$ \\
\hline Testing & 9 & 12 hours & $5 \mathrm{mins}$ \\
\hline
\end{tabular}

\section{$5 \quad$ Experimental Results}

\subsection{Evaluation Measures and Network Architecture}

The performance of the described forecasting methods is measured with two different error functions: Mean Average Error (MAE) and Root Mean Square Error (RMSE) [7]. The error is computed comparing target values for the time series $s_{t+1}, s_{t+2}, \ldots, s_{t+z}$ and its corresponding time series prediction $\hat{\mathrm{s}}_{\mathrm{t}+1}, \hat{\mathrm{s}}_{\mathrm{t}+2}, \ldots, \hat{\mathrm{s}}_{\mathrm{t}+\mathrm{z}}$.

The network architecture consists of an input shape according to the shape of the training data, followed by 32 LSTM blocks and an output layer that makes a single value prediction.

\subsection{Evaluation Results}

The following is a summary of the results based on the above-mentioned measures. Two different models were created, one model was trained with all three mentioned features (sensor signals as described in section 4), the other model only used interior temperature as an input. Table 2 shows the results of the multiple features model, whilst Table 3 presents the results of the single feature model. Changing hyperparameters like learning rate (lr), dropout (do) and recurrent dropout (rdo) had significant impact on MAE and RMSE. 
Table 2. Evaluation measures of LSTM Model with multiple features and different Hyperparameters

\begin{tabular}{|l|l|l|}
\hline Hyperparameter & MAE & RMSE \\
\hline $\mathbf{l r}=\mathbf{0 . 0 0 0 1}, \mathbf{d o}=\mathbf{0 . 1}, \mathbf{r d o}=\mathbf{0 . 1}$ & 0.316055 & 0.383337 \\
\hline $\mathbf{l r}=\mathbf{0 . 0 0 0 1}, \mathbf{d o}=\mathbf{0 . 2}, \mathbf{r d o}=\mathbf{0 . 3}$ & 0.282314 & 0.349757 \\
\hline $\mathbf{l r}=\mathbf{0 . 0 5}, \mathbf{d o}=\mathbf{0 . 1}, \mathbf{r d o}=\mathbf{0 . 1}$ & 0.498570 & 0.637318 \\
\hline
\end{tabular}

Table 3. Evaluation measures of LSTM Model with a single feature and different Hyperparameters

\begin{tabular}{|l|l|l|}
\hline Hyperparameter & MAE & RMSE \\
\hline $\mathbf{l r}=\mathbf{0 . 0 0 0 1}, \mathbf{d o}=\mathbf{0 . 1}, \mathbf{r d o}=\mathbf{0 . 1}$ & 0.308752 & 0.378731 \\
\hline $\mathbf{l r}=\mathbf{0 . 0 0 1}, \mathbf{d o}=\mathbf{0 . 1}, \mathbf{r d o}=\mathbf{0 . 1}$ & 0.223504 & 0.296546 \\
\hline $\mathbf{l r}=\mathbf{0 . 0 5}, \mathbf{d o}=\mathbf{0 . 1}, \mathbf{r d o}=\mathbf{0 . 1}$ & 0.498570 & 0.637318 \\
\hline
\end{tabular}

The training and validation loss (MAE) over 20 epochs corresponding to the best model from Table 3 are displayed in Figure 3. It can be seen that training loss is less than half of validation loss. Training loss seems to improve with the number of epochs while validation loss shows volatility.

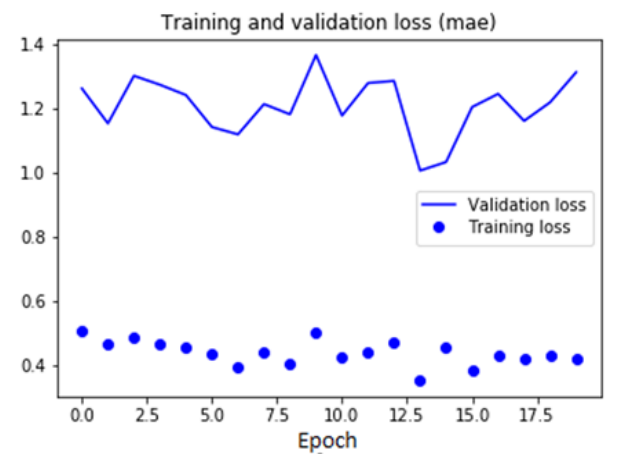

Fig. 3. Training and validation loss (MAE) single feature $(L R=0.001, D O=0.1$, $\mathrm{RDO}=0.1)$

Figure 4 shows the 12-hour prediction of the interior temperature of the best single and multiple feature models presented in Tables 2 and 3. The models are displayed adjoining ground truth. The plot of the single feature model adapts to trends but clearly doesn't show spikes as ground truth. The MAE and RMSE values for the multiple feature model were appropriate but the plot of this model doesn't seem to fit ground truth. The graph doesn't show trends and basically stays within a range of 0.2 degrees Celsius. 


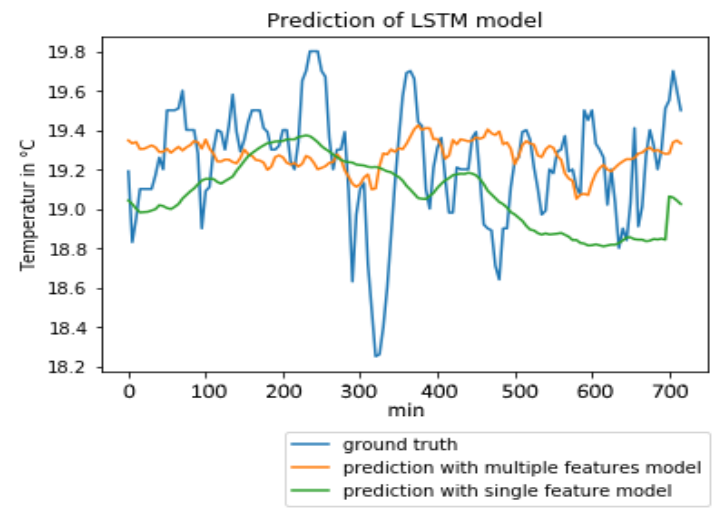

Fig. 4. Prediction of the LSTM model with single and multiple features and ground truth

\section{Conclusion}

The research in this paper has been focused on how to predict the interior temperature of a vehicle (in this case a city bus), as this is directly related to the HVAC system energy consumption. Both a multiple and a single feature model were followed and their performances were examined with MAE and RMSE. The single feature model (only interior temperature) has shown promising results with a MAE $\approx 0.224$ degrees Celsius. The addition of two other features in the multiple feature model didn't show improvements. Air pressure and humidity covariates seem to not add new information to the model in order to improve forecasts.

The results of this work are promising but further research has to be done especially when it comes to the already discussed combination of in-vehicle data with public data. While the overall best result was achieved with only one feature, this is clearly not said for the integration with several other data sources and needs to be examined. For the purpose of generating HVAC energy optimization, these forecasts have to be used for proactive settings of the HVAC system. Usual set points that can be influenced are temperature set point and fan speed. By calculating an optimized HVAC schedule including the time constants of the HVAC control, the potential energy savings need to be evaluated.

\section{References}

1. Traub, M., Vögel, H.J., Sax, E., Streichert, T., Härri, J.: Digitalization in automotive and industrial systems. Proc. 2018 Des. Autom. Test Eur. Conf. Exhib. DATE 2018. 2018January, 1203-1204 (2018). https://doi.org/10.23919/DATE.2018.8342198. 
2. Zamora-Martínez, F., Romeu, P., Botella-Rocamora, P., Pardo, J.: Towards energy efficiency: Forecasting indoor temperature via multivariate analysis. Energies. 6, 4639-4659 (2013). https://doi.org/10.3390/en6094639.

3. Kurdikeri, R.B., Raju, A.B.: Comparative Study of Short-Term Wind Speed Forecasting Techniques Using Artificial Neural Networks. Proc. 2018 Int. Conf. Curr. Trends Towar.

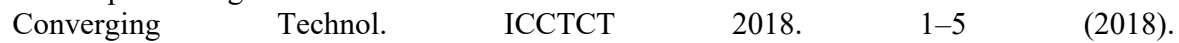
https://doi.org/10.1109/ICCTCT.2018.8550849.

4. Hasim Sak, Andrew Senior, F.B.: Long Short-Term Memory Recurrent Neural Network Architecturesfor Large Scale Acoustic Modeling. INTERSPEECH 2014. (2014). https://doi.org/10.1016/j.edumed.2017.01.001

5. Greff, K., Srivastava, R.K., Koutnik, J., Steunebrink, B.R., Schmidhuber, J.: LSTM: Search Space Odyssey. CoRR. abs/1503.0, 2222-2232 (2015).

6. Massaro, E., Ahn, C., Ratti, C., Santi, P., Stahlmann, R., Lamprecht, A., Roehder, M., Huber, M.: The Car as an Ambient Sensing Platform. Proc. IEEE. 105, 3-7 (2017). https://doi.org/10.1109/JPROC.2016.2634938.

7. Chai, T., Draxler, R.R.: Root mean square error (RMSE) or mean absolute error (MAE)? Arguments against avoiding RMSE in the literature. Geosci. Model Dev. 7, 1247-1250 (2014). https://doi.org/10.5194/gmd-7-1247-2014. 